\title{
2.5D scattering of waves by rigid inclusions buried under a fluid channel via BEM
}

\author{
Julieta António*, António Tadeu, Luís Godinho \\ Department of Civil Engineering, University of Coimbra, Polo II - Pinhal de Marrocos, P-3030-290 Coimbra, Portugal \\ Received 18 October 2004; accepted 7 April 2005
}

Available online 23 May 2005

\begin{abstract}
A 2.5D Boundary Element Method (BEM) formulation, applied in the frequency domain, is developed to compute the scattering of waves by rigid inclusions buried in a semi-infinite solid under a fluid layer, when this system is excited by a spatially-sinusoidal harmonic load.

The BEM algorithm includes Green's functions for a horizontal fluid layer over a semi-infinite solid, which avoids the discretrization of the horizontal surfaces, and thus only the rigid inclusion needs to be discretized by boundary elements. The model uses complex frequencies with a small imaginary part to avoid aliasing phenomena. Time domain responses are obtained by applying an inverse Fourier Transform to the frequency results. The source is modeled as a Ricker pulse. The simulations are performed for three different properties of the solid medium: a fast formation, a slow formation and a sediment formation. (c) 2005 Elsevier SAS. All rights reserved.
\end{abstract}

Keywords: Wave scattering; Rigid inclusions; Solid-fluid interaction; BEM; 2.5D Green's functions

\section{Introduction}

Over the years, different techniques based on wave propagation have been developed to map buried cavities in solids and submerged objects in fluids. They have been supported by a wide variety of analytical and numerical models, implemented to tackle this problem. Some models have also been created to interpret experimental results.

Several studies have been performed using analytical solutions to study the wave scattering caused by cavities (Lee, 1977; Datta and Shah, 1982; Lee, 1988; Lee and Karl, 1992). Recently, Davis et al. (2001) derived analytical solutions to investigate the transverse response of underground cylindrical cavities to incident SV waves. The solutions are derived for unlined cavities embedded in an elastic half space using Fourier-Bessel series and a convex approximation of the half-space free surface. These solutions were extended to formulate approximate solutions for assessing hoop stresses within cavity liners impinged by lowfrequency waves whose wavelengths are much longer than the cavity diameter. They improved the solution proposed by Lee and Karl (1992) by eliminating unwanted reflections on the half-space free surface through a convex approximation.

Guzina and Fataa (2003) used a boundary integral equation method to investigate the problem of mapping three-dimensional underground cavities from surface seismic measurements. The inverse analysis of elastic waves scattered by a three-dimensional void is formulated as a task of minimizing the discrepancy between experimental observations and theoretical predictions for an assumed void geometry.

\footnotetext{
* Corresponding author. Tel.: +351 239797196; fax: +351 239797190.

E-mail address: julieta@dec.uc.pt (J. António).
} 
A boundary integral equation method was formulated by Dawson and Fawcett (1990) to compute the sound scattered underwater by compact deformations of an oceanic waveguide surface. The waveguide surfaces are taken to be flat except for a compact area of deformation where the acoustic scattering takes place. The interaction between the acoustic field and any planar portions of the waveguide are accounted for in the Green's functions so the integrations required to implement the method are restricted to the compact scattering areas.

The same method has been used by Fawcett and Dawson (1990) to compute the three-dimensional pressure field in a waveguide with scattering ridges by solving a sequence of two-dimensional problems. Later, Fawcett (1996a, 1996b) computed the two-dimensional acoustic field scattered by objects embedded between two fluid half-spaces. The method combines the form of the interior cylinder solution with the exterior half-space Green's functions.

Some studies have used methods based on a transition matrix. Kristensson and Ström (1978) presented a three-dimensional formulation using the transition matrix approach to determine the scattering from an inhomogeneity buried in a liquid half space. Boström and Kristensson (1980) subsequently used the same formulation to compute the elastic wave scattering of a cavity buried in a solid half space.

Hackman and Sammelmann (1986) and Sammelmann and Hackman (1987) also used the transition matrix method to analyze the acoustic scattering inside inhomogeneous and homogeneous waveguides in the presence of scatterers. The model requires the host medium to be horizontally stratified and the layer containing the target to be homogeneous.

Lim et al. (1993) used a model based on a full-wave transition matrix implementation of the Helmoltz equation applied to a layered structure to study the scattering of acoustic waves by objects buried in underwater sediments. An object is placed within a thick, planar, homogeneous layer of sediment under deep water. The shear rigidity and the porosity of the sediment are ignored. Lim (1998), also used a transition-matrix solution for the spectral scattering response of a bounded elastic body that penetrates an arbitrary number of layers of a plane-stratified fluid.

Ingenito (1987) proposed an expression for the acoustic field scattered by a rigid sphere in an isovelocity fluid layer overlying a horizontally stratified medium. This is expressed in terms of normal modes and plane-wave scattering functions, and the result is valid when multiple scattering can be disregarded. This model fully accounts for waveguide propagation effects, such as multiple reflections of the scattered field between waveguide boundaries, because it is based on the waveguide Green function.

Makris (1998) described a spectral formulation for 3D object scattering in layered fluid media, valid for receivers and sources placed far enough from the object so that the multiple scattering between the object and the waveguide can be ignored and the scattered field can be written as a linear function of the object's plane-wave scattering function.

Godinho et al. (2001) and Branco et al. (2002) used a boundary element formulation in the frequency domain to study the pressure field generated by point sources placed inside a fluid channel with a rigid deformation on its floor. This model used Green's functions, based on the superposition of virtual sources, to simulate the boundary conditions of the free surface, the rigid flat floor and the lateral walls confining the channel.

In the work described here a BEM formulation in the frequency domain is used to simulate the scattered field produced by a system composed of a rigid inclusion buried in a solid half-space under a fluid channel, when excited by a spatially-sinusoidal harmonic load.

In this problem the geometry is considered constant in the $z$ direction (2D), and so the 3D problem can be written as a summation of 2D problems for varying wavenumbers along this direction.

This model uses Green's functions to simulate a horizontal fluid layer with a free top surface resting over a solid half-space. These analytical solutions for the steady state response of such a formation subjected to a spatially sinusoidal harmonic line load avoid the discretization of the horizontal fluid free surface and the solid fluid interface. In this technique, solid displacement potentials and pressure potentials are used to evaluate the Green's functions for a harmonic (steady-state) line load, with a sinusoidally varying amplitude in the third dimension, in an unbounded medium.

All these displacement potentials are written as a superposition of plane waves, assuming the existence of an infinite number of sources equally spaced along the $x$ direction.

Time domain responses when a rigid circular inclusion is buried in the solid half-space are calculated in the fluid medium for the case where a spatially sinusoidal harmonic line load placed in the fluid medium excites the system.

\section{Green's functions in a fluid layer over a semi-infinite solid medium}

Take a fluid layer with thickness $h$, over a semi-infinite solid medium excited by a spatially sinusoidal harmonic pressure load along the $z$ direction, with frequency $\omega$, acting at the point $\left(x_{0}, y_{0}\right)$ in the fluid or in the solid medium (see Fig. 1).

The Green's functions for a fluid layer, with thickness $h$, over a semi-infinite solid medium can be expressed as the sum of the source terms equal to those in the full-space and the surface terms needed to satisfy the boundary conditions at the free surface of the fluid layer (surface $a$ - null pressures) and at the fluid-solid interface (surface $b$ - continuity of normal displacements and stresses, and null tangential stresses). 


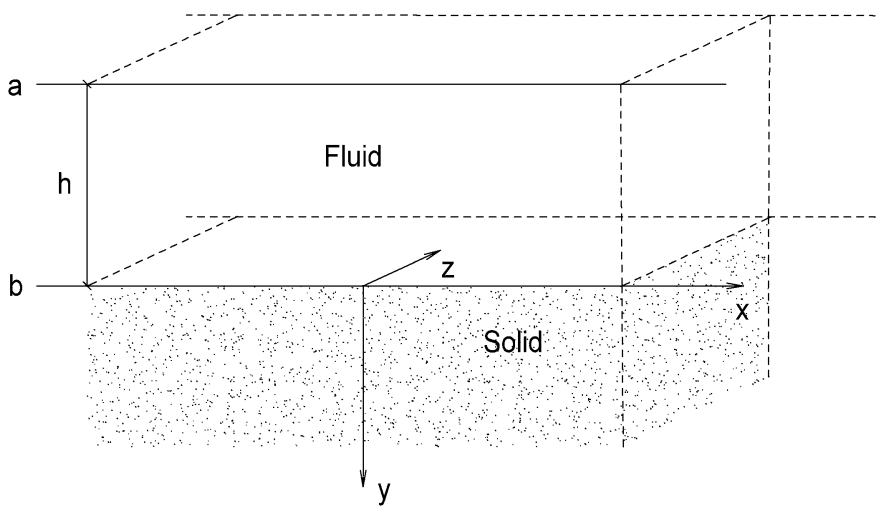

Fig. 1. Scheme of the problem.

The source terms, equal to those in the full-space, are defined as $\sigma_{\text {fluid }}^{\text {full }}$ when the source is placed in the fluid media (pressures due to the incident field in the fluid media) and $G_{i j}^{\text {full }}(i=x, y, z ; j=x, y, z)$ when the source is acting in the solid medium (displacements generated by the incident field in the solid medium). The Green's functions for a full space can be found in Tadeu and Kausel (2000).

The surface terms are defined using solid displacement potentials and fluid pressure potentials, expressed as a superposition of plane waves with different wavenumbers, $k_{n}$, along the $x$ direction. This process adopts the technique used first by Lamb (1904) for the two-dimensional case, and then by Bouchon (1979) and Kim and Papageorgiou (1993) to calculate the threespace dimension field by means of a discrete wave number representation. The problem is formulated assuming the existence of an infinite number of virtual loads distributed along the $x$ direction, at equal intervals $L_{x}$, permitting the definition of $k_{n}=\left(2 \pi / L_{x}\right) n$. The distance $L_{x}$ needs to be large enough to prevent the virtual loads contaminating the response.

In this specific problem, the free surface and the solid-fluid interface generate surface terms, which can be expressed in a form similar to that of the source term.

\subsection{Load in the solid formation acting in the direction of the $x$-axis}

The surface terms generated at the boundaries, when the load acts in the $x$ direction, can be expressed through the following potentials,

Solid medium $(y>0)$

$$
\begin{aligned}
& \phi_{0}^{x}=E_{a} \sum_{n=-N}^{n=+N}\left(\frac{k_{n}}{v_{n}} E_{b 0} A_{n}^{x}\right) E_{d}, \\
& \psi_{x 0}^{x}=0, \\
& \psi_{y 0}^{x}=E_{a} k_{z} \sum_{n=-N}^{n=+N}\left(\frac{E_{c 0}}{\gamma_{n}} B_{n}^{x}\right) E_{d}, \\
& \psi_{z 0}^{x}=-E_{a} \sum_{n=-N}^{n=+N}\left(E_{c 0} C_{n}^{x}\right) E_{d} .
\end{aligned}
$$

Fluid medium $(y<0)$

$$
\begin{aligned}
& \phi_{\text {fluid }}^{b}=-\frac{\mathrm{i}}{L_{x}} \sum_{n=-N}^{n=+N}\left[\left(\frac{-\alpha_{f}^{2}}{\omega^{2} \lambda_{f}}\right) \frac{E_{f 0}}{v_{n}^{f}} D_{n}^{x}\right] E_{d} \quad(\text { surface } b), \\
& \phi_{\text {fluid }}^{a}=-\frac{\mathrm{i}}{L_{x}} \sum_{n=-N}^{n=+N}\left[\left(\frac{-\alpha_{f}^{2}}{\omega^{2} \lambda_{f}}\right) \frac{E_{g 0}}{v_{n}^{f}} E_{n}^{x}\right] E_{d} \quad(\text { surface } a),
\end{aligned}
$$

where $\mathrm{i}=\sqrt{-1}, E_{a}=1 /\left(2 \rho \omega^{2} L_{x}\right), E_{d}=\mathrm{e}^{-\mathrm{i} k_{n}\left(x-x_{0}\right)}, E_{b 0}=\mathrm{e}^{-\mathrm{i} v_{n} y}, E_{c 0}=\mathrm{e}^{-\mathrm{i} \gamma_{n} y}, E_{f 0}=\mathrm{e}^{-\mathrm{i} v_{n}^{f}|y|}, E_{g 0}=\mathrm{e}^{-\mathrm{i} v_{n}^{f}|y+h|}$, $v_{n}=\sqrt{k_{p}^{2}-k_{z}^{2}-k_{n}^{2}}$ with $\operatorname{Im}\left(v_{n}\right) \leqslant 0, \gamma_{n}=\sqrt{k_{s}^{2}-k_{z}^{2}-k_{n}^{2}}$ with $\operatorname{Im}\left(\gamma_{n}\right) \leqslant 0, k_{z}$ is the wavenumber in $z, k_{p}=\omega / \alpha, k_{s}=\omega / \beta$, 
$\alpha=\sqrt{(\lambda+2 \mu) / \rho}$ and $\beta=\sqrt{\mu / \rho}$ are the velocities for $\mathrm{P}$ (pressure) waves and $\mathrm{S}$ (shear) waves, respectively, $\lambda$ and $\mu$ are the Lamé constants, $\rho$ is the mass density in the solid medium; $v_{n}^{f}=\sqrt{k_{p_{f}}^{2}-k_{z}^{2}-k_{n}^{2}}$ with $\operatorname{Im}\left(v_{n}^{f}\right) \leqslant 0, k_{p_{f}}=\omega / \alpha_{f}, \alpha_{f}=$ $\sqrt{\lambda_{f} / \rho_{f}}$ is the acoustic (dilatational) wave velocity of the fluid medium, $\lambda_{f}$ is the fluid Lamé constant and $\rho_{f}$ is the mass density of the fluid.

$A_{n}^{x}, B_{n}^{x}, C_{n}^{x}, D_{n}^{x}$ and $E_{n}^{x}$, are as yet unknown coefficients to be determined from the appropriate boundary conditions, so that the field produced simultaneously by the source and surface terms should produce $\sigma_{y x}^{s}=0, \sigma_{y z}^{s}=0, \sigma_{y y}^{s}=\sigma_{\text {fluid }}^{f}$ and $u_{y}^{s}=u_{y}^{f}$ at $y=0$ and $\sigma_{\text {fluid }}^{f}=0$ at $y=-h$.

Imposing the five stated boundary conditions for each value of $n$ leads to a system of five equations in the five unknown constants. This procedure is quite straightforward, but the details are rather complex, and for this reason are not presented here. The final system of equations is of the form

$$
\left[a_{i j}^{x}, i=1,5 ; j=1,5\right]\left[c_{i}^{x}, i=1,5\right]=\left[b_{i}^{x}, i=1,5\right]
$$

which is fully described in Appendix I.

Once the unknown coefficients have been calculated, the displacements and pressures associated with the surface terms can be obtained using the equations relating the potentials to displacements and pressures. The Green's functions for a solid formation are then obtained from the sum of the source terms and the surface terms originated at the solid-fluid interface. This procedure produces the following expressions for the displacements in the solid formation:

$$
\begin{aligned}
& G_{x x}^{f s}=G_{x x}^{\mathrm{full}}+E_{a} \sum_{n=-N}^{n=+N}\left[A_{n}^{x} \frac{-\mathrm{i} k_{n}^{2}}{v_{n}} E_{b 0}+\left(-\mathrm{i} \gamma_{n} C_{n}^{x}-\frac{\mathrm{i} k_{z}^{2}}{\gamma_{n}} B_{n}^{x}\right) E_{c 0}\right] E_{d}, \\
& G_{x y}^{f s}=G_{x y}^{\mathrm{full}}+E_{a} \sum_{n=-N}^{n=+N}\left(-\mathrm{i} k_{n} A_{n}^{x} E_{b 0}+\mathrm{i} k_{n} C_{n}^{x} E_{c 0}\right) E_{d}, \\
& G_{x z}^{f s}=G_{x z}^{\mathrm{full}}+E_{a} \sum_{n=-N}^{n=+N}\left(\frac{-\mathrm{i} k_{z} k_{n}}{v_{n}} A_{n}^{x} E_{b 0}+\frac{\mathrm{i} k_{z} k_{n}}{\gamma_{n}} B_{n}^{x} E_{c 0}\right) E_{d},
\end{aligned}
$$

$G_{i j}^{\text {full }}(i, j=x, y, z)$ are the displacements for the full space.

The final expression for the pressure field in the fluid medium is then given by the sum of the surface terms for pressures originated in the fluid layer boundaries

$$
\sigma_{\text {fluid }}^{x}=-\frac{\mathrm{i}}{L_{x}}\left[\sum_{n=-N}^{n=+N}\left(\frac{k_{n} E_{f 0}}{v_{n}^{f}} D_{n}^{x}\right)+\sum_{n=-N}^{n=+N}\left(\frac{k_{n} E_{f 0}^{b}}{v_{n}^{f}} E_{n}^{x}\right)\right] E_{d} \quad(\text { when } y<0) .
$$

The expressions for forces applied along the $y$ and $z$ directions can be derived in the same way.

Note that if $k_{z}=0$ is used, the system of equations derived above is reduced to four unknowns, leading to the twodimensional Green's function for plane strain line-loads. This procedure is repeated, if $k_{z}=0$, for all the load cases described next.

\subsection{Load in the solid formation acting in the direction of the $y$-axis}

The surface terms generated at the two interfaces can be expressed through the following potentials,

Solid medium $(y>0)$

$$
\begin{aligned}
& \phi_{0}^{y}=E_{a} \sum_{n=-N}^{n=+N}\left(E_{b 0} A_{n}^{y}\right) E_{d}, \\
& \psi_{x 0}^{y}=E_{a} k_{z} \sum_{n=-N}^{n=+N}\left(\frac{-E_{c 0}}{\gamma_{n}} C_{n}^{y}\right) E_{d}, \\
& \psi_{y 0}^{y}=0, \\
& \psi_{z 0}^{y}=E_{a} \sum_{n=-N}^{n=+N}\left(\frac{k_{n}}{\gamma_{n}} E_{c 0} B_{n}^{y}\right) E_{d} .
\end{aligned}
$$


Fluid medium $(y<0)$

$$
\begin{aligned}
& \left.\phi_{\text {fluid }}^{b}=-\frac{\mathrm{i}}{L_{x}} \sum_{n=-N}^{n=+N}\left[\left(\frac{-\alpha_{f}^{2}}{\omega^{2} \lambda_{f}}\right) \frac{E_{f 0}}{v_{n}^{f}} D_{n}^{y}\right] E_{d} \quad \text { (surface } b\right), \\
& \phi_{\text {fluid }}^{a}=-\frac{\mathrm{i}}{L_{x}} \sum_{n=-N}^{n=+N}\left[\left(\frac{-\alpha_{f}^{2}}{\omega^{2} \lambda_{f}}\right) \frac{E_{g 0}}{v_{n}^{f}} E_{n}^{y}\right] E_{d} \quad(\text { surface } a) .
\end{aligned}
$$

The imposition of the five stated boundary conditions for each value of $n$ leads to a system of five equations in the five unknown constants,

$$
\left[a_{i j}^{y}, i=1,5 ; j=1,5\right]\left[c_{i}^{y}, i=1,5\right]=\left[b_{i}^{y}, i=1,5\right]
$$

which is fully described in Appendix II.

Once the amplitude of each potential has been calculated, the Green's functions for the displacements in the solid formation are then given by the sum of the source terms and the surface terms originated at the solid-fluid interface,

$$
\begin{aligned}
& G_{y x}^{f s}=G_{y x}^{\mathrm{full}}+E_{a} \sum_{n=-N}^{n=+N}\left(-\mathrm{i} k_{n} \operatorname{sgn}(y) A_{n}^{y} E_{b 0}+\mathrm{i} k_{n} \operatorname{sgn}(y) B_{n}^{y} E_{c 0}\right) E_{d}, \\
& G_{y y}^{f s}=G_{y y}^{\mathrm{full}}+E_{a} \sum_{n=-N}^{n=+N}\left[-\mathrm{i} v_{n} A_{n}^{y} E_{b 0}+\left(\frac{-\mathrm{i} k_{n}^{2}}{\gamma_{n}} B_{n}^{y}+\frac{-\mathrm{i} k_{z}^{2}}{\gamma_{n}} C_{n}^{y}\right) E_{c 0}\right] E_{d}, \\
& G_{y z}^{f s}=G_{y z}^{\mathrm{full}}+E_{a} \sum_{n=-N}^{n=+N}\left(-\mathrm{i} k_{z} \operatorname{sgn}(y) A_{n}^{y} E_{b 0}+\mathrm{i} k_{z} \operatorname{sgn}(y) C_{n}^{y} E_{c 0}\right) E_{d} .
\end{aligned}
$$

The final expression for the pressure field in the fluid medium is then given by the sum of the surface terms originated in the fluid interfaces $a$ and $b$

$$
\sigma_{\text {fluid }}^{y}=-\frac{\mathrm{i}}{L_{x}}\left[\sum_{n=-N}^{n=+N}\left(\frac{E_{f 0}}{v_{n}^{f}} D_{n}^{y}\right)+\sum_{n=-N}^{n=+N}\left(\frac{E_{f 0}^{b}}{v_{n}^{f}} E_{n}^{y}\right)\right] E_{d} \quad(\text { when } y<0) .
$$

\subsection{Load in the solid formation acting in the direction of the $z$-axis}

The surface terms generated at the two interfaces can be expressed using the following potentials, which have been derived using the technique described above,

Solid medium $(y>0)$

$$
\begin{aligned}
& \phi_{0}^{z}=E_{a} k_{z} \sum_{n=-N}^{n=+N}\left(\frac{E_{b 0}}{v_{n}} A_{n}^{z}\right) E_{d}, \\
& \psi_{x 0}^{z}=E_{a} \sum_{n=-N}^{n=+N}\left(E_{c 0} B_{n}^{z}\right) E_{d}, \\
& \psi_{y 0}^{z}=E_{a} \sum_{n=-N}^{n=+N}\left(\frac{-k_{n}}{\gamma_{n}} E_{c 0} C_{n}^{z}\right) E_{d}, \\
& \psi_{z 0}^{z}=0 .
\end{aligned}
$$

Fluid medium $(y<0)$

$$
\begin{aligned}
& \phi_{\text {fluid }}^{b}=-\frac{\mathrm{i}}{L_{x}} \sum_{n=-N}^{n=+N}\left[\left(\frac{-\alpha_{f}^{2}}{\omega^{2} \lambda_{f}}\right) \frac{E_{f 0}}{v_{n}^{f}} D_{n}^{z}\right] E_{d} \quad(\text { surface } b), \\
& \phi_{\text {fluid }}^{a}=-\frac{\mathrm{i}}{L_{x}} \sum_{n=-N}^{n=+N}\left[\left(\frac{-\alpha_{f}^{2}}{\omega^{2} \lambda_{f}}\right) \frac{E_{g 0}}{v_{n}^{f}} E_{n}^{z}\right] E_{d} \quad(\text { surface } a) .
\end{aligned}
$$


The imposition of the five stated boundary conditions for each value of $n$ leads to a system of five equations in the five unknown constants,

$$
\left[a_{i j}^{z}, i=1,5 ; j=1,5\right]\left[c_{i}^{z}, i=1,5\right]=\left[b_{i}^{z}, i=1,5\right]
$$

which is fully described in Appendix III.

Once the unknown amplitude of each potential has been calculated, the Green's functions for the solid formation are given by the sum of the source terms and the surface terms originated at the fluid-solid interface, leading to the following expressions,

$$
\begin{aligned}
& G_{z x}^{f s}=G_{z x}^{\mathrm{full}}+E_{a} \sum_{n=-N}^{n=+N}\left(\frac{-\mathrm{i} k_{z} k_{n}}{v_{n}} A_{n}^{z} E_{b 0}+\frac{\mathrm{i} k_{z} k_{n}}{\gamma_{n}} C_{n}^{z} E_{c 0}\right) E_{d}, \\
& G_{z y}^{f s}=G_{z y}^{\mathrm{full}}+E_{a} \sum_{n=-N}^{n=+N}\left(-\mathrm{i} k_{z} \operatorname{sgn}(y) A_{n}^{z} E_{b 0}+\mathrm{i} k_{z} \operatorname{sgn}(y) B_{n}^{z} E_{c 0}\right) E_{d}, \\
& G_{z z}^{f s}=G_{z z}^{\mathrm{full}}+E_{a} \sum_{n=-N}^{n=+N}\left[\frac{-\mathrm{i} k_{z}^{2}}{v_{n}} A_{n}^{z} E_{b 0}+\left(\frac{-\mathrm{i} k_{n}^{2}}{\gamma_{n}} C_{n}^{z}-\mathrm{i} \gamma_{n} B_{n}^{z}\right) E_{c 0}\right] E_{d} .
\end{aligned}
$$

The final expression for the pressure field in the fluid media is given by the sum of the surface terms originated in fluid layer interfaces,

$$
\sigma_{\text {fluid }}^{z}=-\frac{\mathrm{i}}{L_{x}}\left[\sum_{n=-N}^{n=+N}\left(\frac{E_{f 0}}{v_{n}^{f}} D_{n}^{z}\right)+\sum_{n=-N}^{n=+N}\left(\frac{E_{f 0}^{b}}{v_{n}^{f}} E_{n}^{z}\right)\right] E_{d} \quad(\text { when } y<0) .
$$

\subsection{Pressure load acting in the fluid layer}

The surface terms produced at the horizontal interfaces can be expressed using the following potentials,

Solid medium $(y>0)$

$$
\begin{aligned}
& \phi_{0}^{f}=E_{a} \sum_{n=-N}^{n=+N}\left(E_{b 0} A_{n}^{f}\right) E_{d}, \\
& \psi_{x 0}^{f}=E_{a} k_{z} \sum_{n=-N}^{n=+N}\left(\frac{-E_{c 0}}{\gamma_{n}} C_{n}^{f}\right) E_{d}, \\
& \psi_{y 0}^{f}=0, \\
& \psi_{z 0}^{f}=E_{a} \sum_{n=-N}^{n=+N}\left(\frac{k_{n}}{\gamma_{n}} E_{c 0} B_{n}^{f}\right) E_{d} .
\end{aligned}
$$

Fluid medium $(y<0)$

$$
\begin{aligned}
& \phi_{\text {fluid }}^{b}=-\frac{\mathrm{i}}{L_{x}} \sum_{n=-N}^{n=+N}\left[\left(\frac{-\alpha_{f}^{2}}{\omega^{2} \lambda_{f}}\right) \frac{E_{f 0}}{v_{n}^{f}} D_{n}^{f}\right] E_{d} \quad(\text { surface } b), \\
& \phi_{\text {fluid }}^{a}=-\frac{\mathrm{i}}{L_{x}} \sum_{n=-N}^{n=+N}\left[\left(\frac{-\alpha_{f}^{2}}{\omega^{2} \lambda_{f}}\right) \frac{E_{g 0}}{v_{n}^{f}} E_{n}^{f}\right] E_{d} \quad(\text { surface } a) .
\end{aligned}
$$

After the five stated boundary conditions, for each value of $n$, have been imposed, a system of five equations in the five unknown constants is built up,

$$
\left[a_{i j}^{f}, i=1,5 ; j=1,5\right]\left[c_{i}^{f}, i=1,5\right]=\left[b_{i}^{f}, i=1,5\right]
$$

details of which are given in Appendix IV.

After the system of equations has been solved, the Green's functions for the solid formation are given by the surface terms originated at the fluid-solid interface, generating the following expressions, 


$$
\begin{aligned}
& G_{f x}^{f s}=E_{a} \sum_{n=-N}^{n=+N}\left(-\mathrm{i} k_{n} \operatorname{sgn}(y) A_{n}^{f} E_{b 0}+\mathrm{i} k_{n} \operatorname{sgn}(y) B_{n}^{f} E_{c 0}\right) E_{d}, \\
& G_{f y}^{f s}=E_{a} \sum_{n=-N}^{n=+N}\left[-\mathrm{i} v_{n} A_{n}^{f} E_{b 0}+\left(\frac{-\mathrm{i} k_{n}^{2}}{\gamma_{n}} B_{n}^{f}+\frac{-\mathrm{i} k_{z}^{2}}{\gamma_{n}} C_{n}^{f}\right) E_{c 0}\right] E_{d}, \\
& G_{f z}^{f s}=E_{a} \sum_{n=-N}^{n=+N}\left(-\mathrm{i} k_{z} \operatorname{sgn}(y) A_{n}^{f} E_{b 0}+\mathrm{i} C_{n}^{f} k_{z} E_{c 0}\right) E_{d} .
\end{aligned}
$$

The final expression for the pressure field in the fluid medium is then given by the addition of the pressure source term and the surface terms originated at the fluid layer interfaces,

$$
\sigma_{\text {fluid }}^{f}=\sigma_{\text {fluid }}^{\text {full }}-\frac{\mathrm{i}}{L_{x}}\left[\sum_{n=-N}^{n=+N}\left(\frac{E_{f 0}}{v_{n}^{f}} D_{n}^{f}\right)+\sum_{n=-N}^{n=+N}\left(\frac{E_{f 0}^{b}}{v_{n}^{f}} E_{n}^{f}\right)\right] E_{d} \quad(\text { when } y<0)
$$

$\sigma_{\text {fluid }}^{\text {full }}=-\mathrm{i} / 2 H_{0}\left(\sqrt{\left(k_{p f}\right)^{2}-\left(k_{z}\right)^{2}}\right)$ is the expression for pressure in the full space fluid medium. In this expression $H_{0}()$ is the second Hankel function of order 0 .

\section{BEM formulation}

Consider that the horizontal fluid layer with a free top surface, bounded by a semi-infinite solid elastic medium, defined above, contains now a rigid inclusion. This system is subjected to the 3D wavefield generated by a spatially sinusoidal harmonic line load located in the fluid medium.

The Boundary Element Method (BEM) is suitable for solving this problem. The use of Green's functions for an unbounded medium would require the discretization of all interfaces, which would lead to a very demanding computing effort.

The BEM formulation used here employs the Green's functions derived above, for a horizontal fluid layer over a semiinfinite solid medium. Thus, only the rigid boundary of the inclusion, requiring null displacement boundary conditions, needs to be discretized. In these conditions the integral equation to be applied to the rigid boundary in the presence of an incident wave is

$$
\int_{C} t_{j}(\boldsymbol{x}, v, \omega) G_{i j}^{f s}\left(\boldsymbol{x}, \boldsymbol{x}_{0}, \omega\right) \mathrm{d} s+G_{f i}^{f s(\text { inc })}\left(\boldsymbol{x}_{0}, \omega\right)=0
$$

where, $i, j=1,2$ are the normal and tangential directions in relation to the boundary surface; $i, j=3$ indicates the $z$ direction; $G_{i j}^{f s}\left(\boldsymbol{x}, \boldsymbol{x}_{0}, \omega\right)$, are the Green's function displacements in direction $j$ at $\boldsymbol{x}$, on boundary $C$, originated by a unit sinusoidal line load acting at the source point, $\boldsymbol{x}_{0}$, in direction $i$; vector $v$ is the unit outward normal at the boundary, $t_{j}(\boldsymbol{x}, v, \omega)$ are the tractions to be determined in the boundary. The incident field in this equation is given analytically as the surface terms originated on both horizontal surfaces $G_{f i}^{f s(\text { inc) }}\left(\boldsymbol{x}_{0}, \omega\right)$ (see Eq. (19)).

The boundary needs to be discretized before this integral can be evaluated for an arbitrary cross-section. The boundary is discretized into $N$ straight boundary elements, with constant interpolation functions and one nodal point in the middle of each element.

To verify the accuracy of the results provided by this model, a BEM model which uses Green's functions for unbounded medium was built. Three different boundaries need to be discretized: the top of the fluid layer where the required boundary conditions are null pressures; the solid-fluid interface where continuity of normal tractions and normal displacements, and null tangential stresses, need to be established; and the boundary of the rigid inclusion for which null displacements are required. Thus, the following boundary integral equations are required:

(a) along the bottom surface of the fluid layer

$$
c p(\boldsymbol{x}, \nu, \omega)=\int_{C} p(\boldsymbol{x}, \omega) G_{f 1}^{\mathrm{full}}\left(\boldsymbol{x}, \boldsymbol{x}_{0}, \omega\right) \mathrm{d} s-\int_{C} H_{f 1}^{\mathrm{full}}\left(\boldsymbol{x}, v, \boldsymbol{x}_{0}, \omega\right) u_{1}(\boldsymbol{x}, v, \omega) \mathrm{d} s+p^{\mathrm{inc}}(\boldsymbol{x}, \omega),
$$

where $G_{f 1}^{\text {full }}\left(\boldsymbol{x}, \boldsymbol{x}_{0}, \omega\right)$ and $H_{f 1}^{\text {full }}\left(\boldsymbol{x}, v, \boldsymbol{x}_{0}, \omega\right)$ are the Green's functions for displacements and for pressures respectively; $u_{1}(\boldsymbol{x}, v, \omega)$ and $p(\boldsymbol{x}, \omega)$ are normal displacements and pressures on the boundary, $c$ is a constant depending on the boundary geometry. $p^{\text {inc }}(\boldsymbol{x}, \omega)=\sigma_{\text {fluid }}^{\text {full }}$ is the incident pressure field. 
(b) Along the top surface of the fluid layer

$$
c p(\boldsymbol{x}, v, \omega)=-\int_{C} H_{f 1}^{\mathrm{full}}\left(\boldsymbol{x}, v, \boldsymbol{x}_{0}, \omega\right) u_{1}(\boldsymbol{x}, v, \omega) \mathrm{d} s+p^{\mathrm{inc}}(\boldsymbol{x}, \omega) .
$$

(c) Along the solid horizontal interface

$$
c_{i j} u_{i}\left(\boldsymbol{x}_{0}, \omega\right)=\int_{C} t_{j}(\boldsymbol{x}, v, \omega) G_{i j}^{\mathrm{full}}\left(\boldsymbol{x}, \boldsymbol{x}_{0}, \omega\right) \mathrm{d} s-\int_{C} H_{i j}^{\mathrm{full}}\left(\boldsymbol{x}, v, \boldsymbol{x}_{0}, \omega\right) u_{j}(\boldsymbol{x}, \omega) \mathrm{d} s,
$$

where $G_{i j}^{\text {full }}\left(\boldsymbol{x}, \boldsymbol{x}_{0}, \omega\right)$ and $H_{i j}^{\text {full }}\left(\boldsymbol{x}, v, \boldsymbol{x}_{0}, \omega\right)$ are Green's functions for displacements and tractions in an unbounded medium, respectively.

(d) Along the boundary of the rigid inclusion

$$
\int_{C} t_{j}(\boldsymbol{x}, v, \omega) G_{i j}^{\mathrm{full}}\left(\boldsymbol{x}, \boldsymbol{x}_{0}, \omega\right) \mathrm{d} s=0 .
$$

The unlimited discretization of the top and bottom fluid layer surfaces in this BEM model is accomplished by using complex frequencies with a small imaginary part of the form $\omega_{c}=\omega-\mathrm{i} \eta$ (with $\eta=7.5(2 \pi / T)$ ), which introduces a damping effect.

\section{Verification of the solution}

The two models were compared computing the results for a water layer $\left(\rho_{f}=1000 \mathrm{~kg} / \mathrm{m}^{3}, \alpha_{f}=1500 \mathrm{~m} / \mathrm{s}\right), 10.0 \mathrm{~m}$ thick, over a slow formation $\left(\rho=2250 \mathrm{~kg} / \mathrm{m}^{3}, \alpha=2630 \mathrm{~m} / \mathrm{s}, \beta=1416 \mathrm{~m} / \mathrm{s}\right)$ solid medium in which a circular rigid inclusion with radius $3.0 \mathrm{~m}$ is buried, centered at $x=0.0 \mathrm{~m}$ and $y=8.0 \mathrm{~m}$. The calculations are performed in the frequency domain $[2.0,320.0 \mathrm{~Hz}]$ with a frequency increment of $2.0 \mathrm{~Hz}$. The response is computed for a single value of $k_{z}\left(k_{z}=0.4 \mathrm{rad} / \mathrm{m}\right)$. The BEM model using Green's functions for an unbounded medium required the use of 560 boundary elements distributed along the boundaries. The limitation of the discretized horizontal boundaries $(220 \mathrm{~m})$ was achieved using complex frequencies with a large amount of damping. Only 50 boundary elements were used to model the inclusion when the BEM model incorporated the proposed Green's functions.

To illustrate the agreement between the two solutions, only the pressures are displayed at receiver $\mathrm{R} 1 \mathrm{placed}$ at $x=2.0 \mathrm{~m}$ and $y=-2.0 \mathrm{~m}$ (in the fluid), and displacements along the $y$ direction at R2 placed at $x=2.0 \mathrm{~m}$ and $y=3.0 \mathrm{~m}$ (in the solid), when the source is acting in the fluid at $(x=0.0 \mathrm{~m}$ and $y=-1.0 \mathrm{~m})$ or when the source is acting in the solid medium along the $y$ direction.

Fig. 2(a) presents the real and imaginary parts of the scattered pressure field recorded at a receiver R1 while Fig. 2(b) shows the scattered displacements in the $y$ direction recorded at a receiver R2 when the source is placed in the fluid.

Fig. 3(a) shows the real and imaginary parts of the scattered pressure field recorded at a receiver R1 and Fig. 3(b) exhibits the scattered displacements in the $y$ direction recorded at a receiver $\mathrm{R} 2$ when the source is placed in the solid medium.

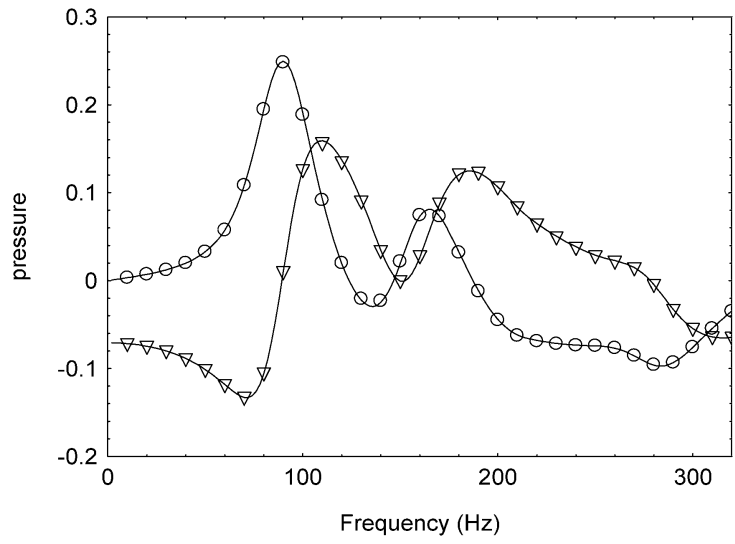

(a)

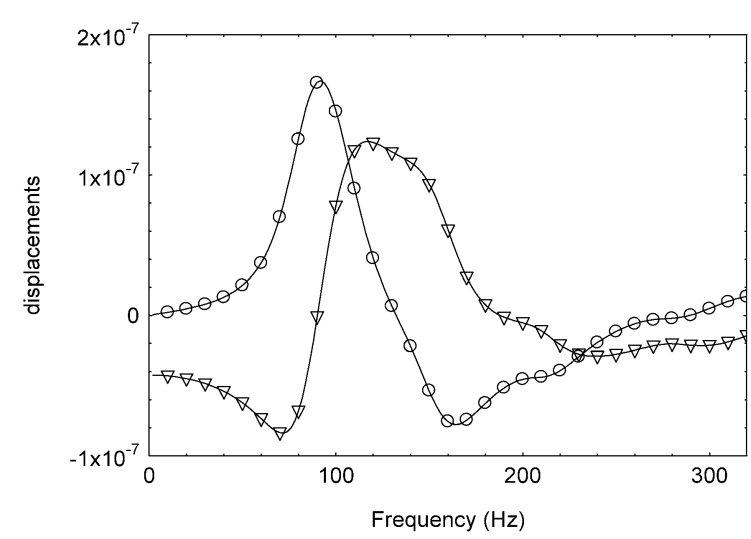

(b)

Fig. 2. Load in the fluid medium: (a) pressures at receiver R1; (b) displacements along the $y$ direction at receiver R2. 


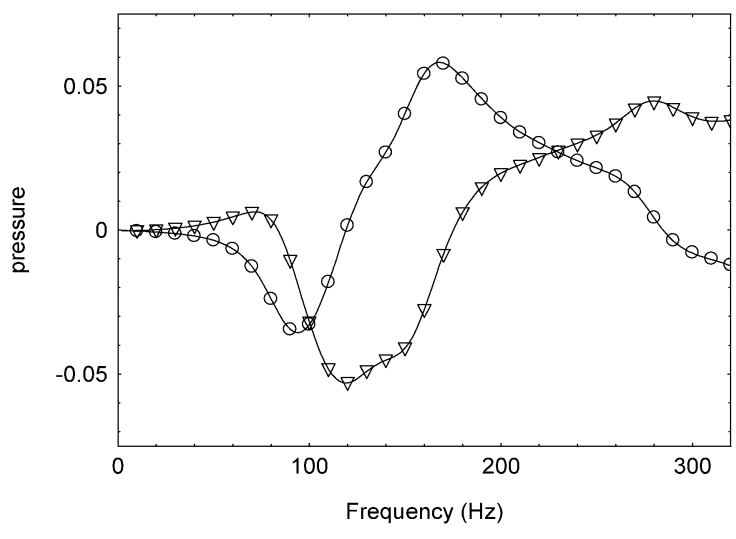

(a)

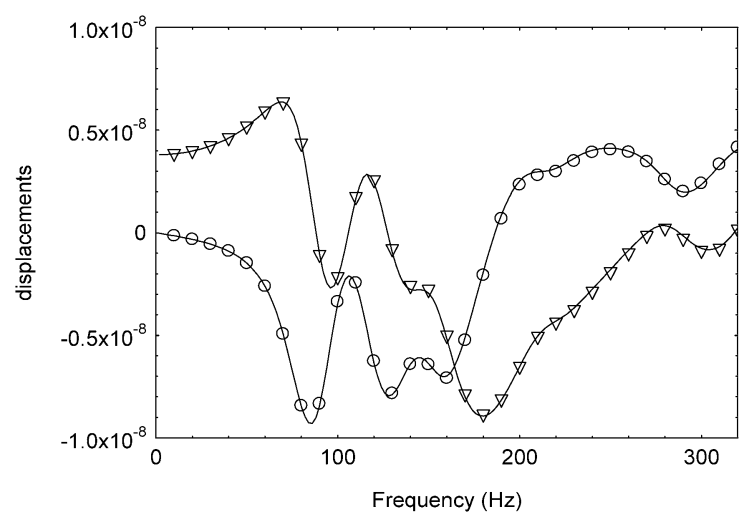

(b)

Fig. 3. Load in the solid medium acting along the $y$ direction: (a) pressures at receiver R1; (b) displacements along the $y$ direction at receiver R2.

Table 1

Properties of the solid formations

\begin{tabular}{llll}
\hline & Compressional wave velocity $(\mathrm{m} / \mathrm{s})$ & Shear wave velocity $(\mathrm{m} / \mathrm{s})$ & Density $\left(\mathrm{kg} / \mathrm{m}^{3}\right)$ \\
\hline Fast formation & $\alpha=4208$ & $\beta=2656$ & $\rho=2140$ \\
Slow formation & $\alpha=2630$ & $\beta=1416$ & $\rho=2250$ \\
Sediment & $\alpha=1643$ & $\beta=526$ & $\rho=1590$ \\
\hline
\end{tabular}

The solid lines represent the results calculated using the BEM model, which includes the Green's functions presented in this paper, while the marked points represent the BEM solution using Green's functions for an unbounded medium. The triangular marks correspond to the real part of the response, while the round marks represent the imaginary part. The results reveal an excellent agreement between the two solutions.

It should be noted that in the vicinity of the eigenfrequencies of the system, the BEM solution may not be so accurate. A sufficiently fine boundary discretization and the use of damping reduces the probability of observing that behaviour.

\section{Numerical applications}

The model described above was used to compute the pressure field in a fluid layer over a semi-infinite solid formation where a rigid circular inclusion is buried. The numerical applications are performed assuming a water layer $\left(\rho_{f}=1000 \mathrm{~kg} / \mathrm{m}^{3}\right.$, $\alpha_{f}=1500 \mathrm{~m} / \mathrm{s}$ ), $20.0 \mathrm{~m}$ thick, over different elastic solid formations with properties (listed at Table 1) corresponding to a fast formation $\left(\beta<\alpha_{f}\right)$, a slow formation $\left(\beta>\alpha_{f}\right)$ or a sediment formation.

The rigid inclusion is centered at $x=0.0 \mathrm{~m}$ and $y=5.0 \mathrm{~m}$ (see Fig. 4) with a radius of $2.0 \mathrm{~m}$. The boundary of the inclusion is discretized using a number of constant boundary elements defined according to the excitation frequency of the harmonic source. The ratio between the wavelength of the incident waves and the length of the boundary elements is kept to a minimum of 6 . However, the number of boundary elements used to model the inclusion was never less than 30.

The system is perturbed by a pressure line load $\left(k_{z}=0.0 \mathrm{rad} / \mathrm{m}\right)$ placed in the water medium at $x=-20.0 \mathrm{~m}$ and $y=-1.0 \mathrm{~m}$. The pressure variations in the fluid are registered at a grid of 61 by 40 receivers placed along the $x$ and $y$ direction respectively. The receivers are separated by $0.5 \mathrm{~m}$ in either direction and are placed from $x=-15.0 \mathrm{~m}$ and $y=-0.25 \mathrm{~m}$ to $x=15.0 \mathrm{~m}$ and $y=-19.75 \mathrm{~m}$ (see Fig. 4).

All the computations are carried out in the frequency domain using complex frequencies of the form $\omega_{c}=\omega-\mathrm{i} \eta$ (with $\eta=0.7 \Delta \omega$ ) to prevent aliasing phenomena. The frequency range used is [10 Hz, $1280 \mathrm{~Hz}]$ with a frequency increment of $10 \mathrm{~Hz}$. This increment defines the total time duration of $100 \mathrm{~ms}$. An inverse Fourier transform is then applied to the frequency results in order to obtain time domain responses. The source is modeled as a Ricker pulse with a characteristic frequency of $350 \mathrm{~Hz}$.

The time pressure amplitude registered along the lower line of receivers is presented in order to identify the different type of waves. A sequence of snapshots that displays the pressure wave field along the grid of receivers at different times is also displayed. The pressure amplitude is plotted in a gray scale ranging from black to white, as the amplitude increases. 


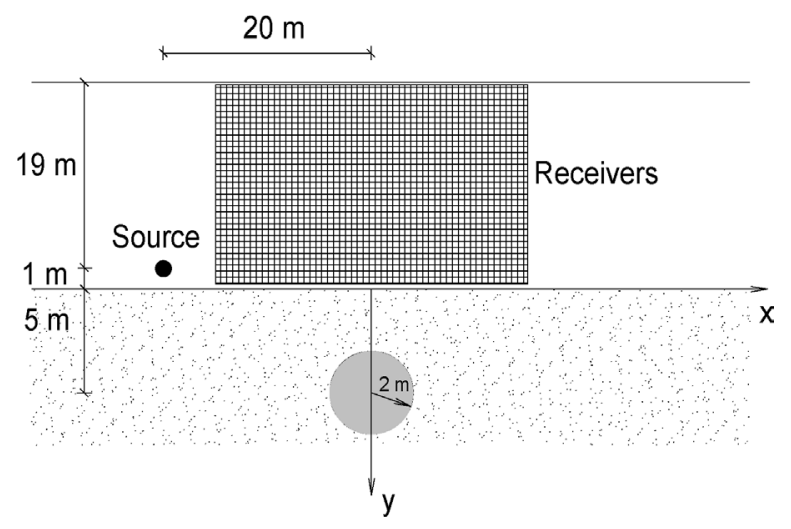

Fig. 4. Geometry of the model.

Fig. 5 presents the time results when the solid elastic medium has the properties of the fast formation. At $t=0.0 \mathrm{~s}$ the source emits a pulse that propagates away, giving rise to a spread of energy. As time elapses the incident pulse hits the fluid solid interface and the water free surface leading to a complex wavefield. However, it is possible to identify waves reflecting back from the free surface, waves propagating along the fluid-solid interface with the velocity of the $\mathrm{S}$ waves in the solid $(2656 \mathrm{~m} / \mathrm{s})$, and waves transmitted to the solid medium that are then reflected back by the rigid cavity.

Fig. 5(a) gives the time pressures registered along the lower line of receivers, while Figs. 5(b)-(e) show snapshots computed over the grid of receivers at different times. In Fig. 5(a) different types of pulses are distinguishable. The first pulse (FPF), with a weak amplitude, corresponds to waves which, once they reach the fluid-solid interface, propagate along it with the velocity of the $\mathrm{P}$ waves in the solid $(4208 \mathrm{~m} / \mathrm{s})$. On a different plot scale this pulse would be visible in the snapshot at $t=7.5 \mathrm{~ms}$, at $x=5.0 \mathrm{~m}$. A pulse with higher amplitude follows, labelled FSF. This pulse corresponds to waves which, after hitting the fluid-solid interface, propagate with the velocity of the $\mathrm{S}$ waves in the solid $(2656 \mathrm{~m} / \mathrm{s})$. The direct incident pulse (I) appears later, since it propagates at lower velocity (velocity of the fluid, $1500 \mathrm{~m} / \mathrm{s}$ ). This pulse is visible in the snapshots for $t=7.5 \mathrm{~ms}$, $t=12.5 \mathrm{~ms}$ and $t=20 \mathrm{~ms}$ (Figs. 5(b)-(d)). Reflections from the cavity are also present. One of the most evident corresponds to waves that are transmitted to the solid medium and are reflected by the rigid boundary of the inclusion. It propagates in the solid medium with the velocity of the $\mathrm{S}$ waves (FSSF). This pulse is also visible in the snapshot for $t=12.5 \mathrm{~ms}$. As time elapses, the wave front hits the free water surface, where is reflected back. These waves reach the fluid-solid interface where they are again reflected back, and this process continues. These reflections are identified in Fig. 5(a) by FF and FFFF. Figs. 5(d) and 5(e) show the FF pulse being reflected at the top and bottom interfaces.

Fig. 6 presents the pressure field obtained for the case of a slow formation. Fig. 6(a) shows the time results obtained for the lower line of receivers (at $y=0.25 \mathrm{~m}$ ), while Figs. 6(b)-(e) display the pressure responses registered at the grid of receivers at specific times.

The faster pulse (FPF) is associated with waves which, after the incident pulse has reached the solid-fluid interface, propagate along it with the velocity of $\mathrm{P}$ waves $(2630 \mathrm{~m} / \mathrm{s})$ in the solid. This pulse is visible in the snapshots at $t=7.5 \mathrm{~ms}$ and $t=12.5 \mathrm{~ms}$ (Figs. 6(b) and 6(c) respectively). A pulse that is transmitted to the solid with the velocity of the P waves and propagates with the velocity of the $\mathrm{S}$ waves after being reflected back by the rigid inclusion is identified by FPSF (see Figs. 6(a), 6(c)). A well-developed pulse follows, related to the direct incident waves travelling with the velocity of the fluid $(1500 \mathrm{~m} / \mathrm{s})$. Another high-amplitude pulse can be seen in Fig. 6(a). This pulse corresponds to guided waves (G) travelling along the interface with velocities lower than the S wave velocity. As can be seen in Figs. 6(c)-(e), this pulse is only registered at receivers near the interface. As the wave propagation progresses additional reflections arise, such as waves propagating in the solid with the velocity of the S waves that are scattered by the rigid inclusion (FSSF). Additional reflections are visible, corresponding to waves propagating back and forth in the fluid medium between the free surface and the solid-fluid interface (FF, FFFF). High order reflections at the boundary of the rigid cavity are also present.

Next, we give some results when the solid medium under the fluid layer is sediment. Fig. 7 shows the pressure field registered at the line of receivers in the time domain, and snapshots of the pressure field registered at the grid of receivers for different times.

As before, the pressure field is made up of different types of waves. It is possible to observe the direct incident waves (I) which, once they reach the solid fluid interface, propagate along it with the velocity of the $P$ waves $(1643 \mathrm{~m} / \mathrm{s})$ in the solid (FPF) (see Fig. 7(a)). These two pulses are not easily distinguishable since the velocity in the fluid medium $(1500 \mathrm{~m} / \mathrm{s})$ approaches the velocity of the $\mathrm{P}$ waves in the solid. A well-developed pulse (FPPF), corresponding to the first reflections by the rigid inclusion of waves propagating in the solid medium with the velocity of the $\mathrm{P}$ waves, is also visible. 


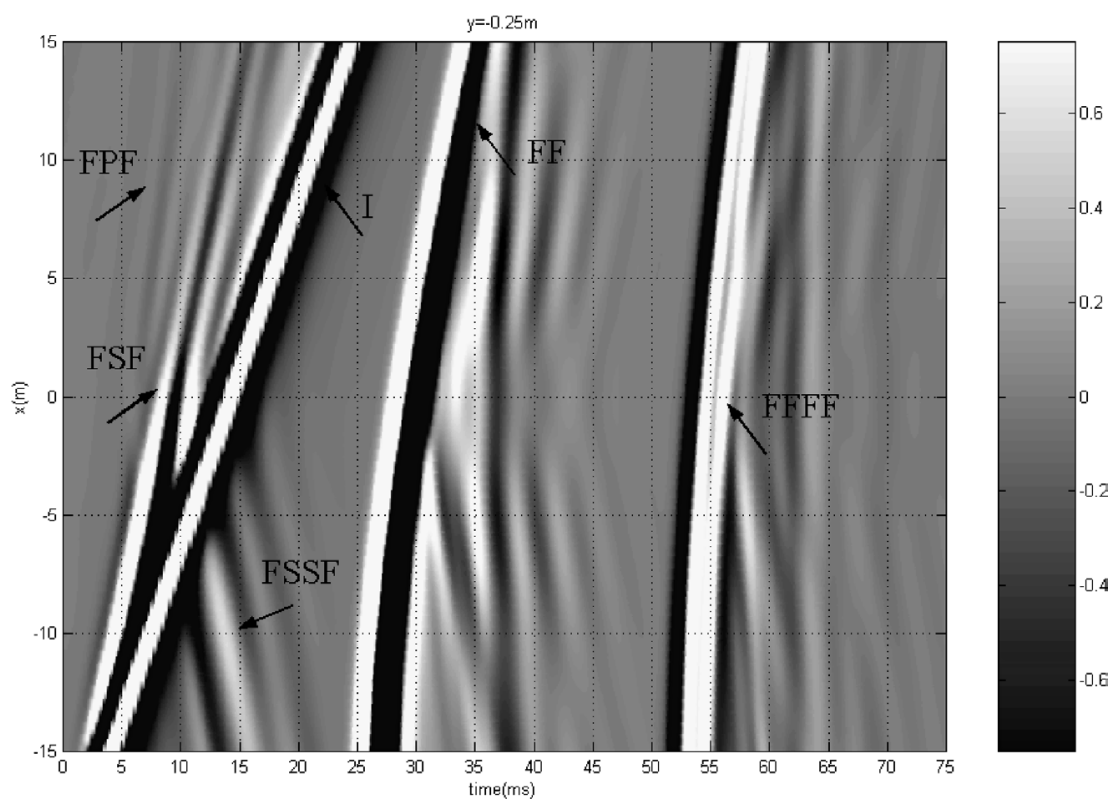

(a)

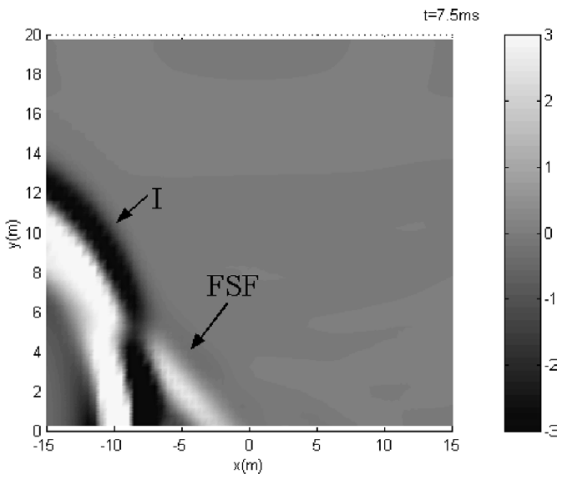

(b)

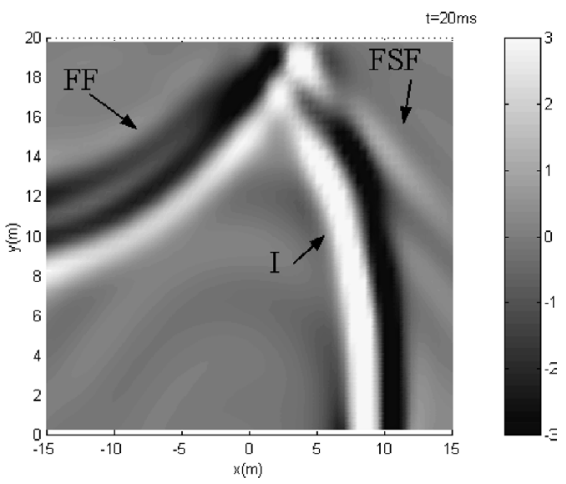

(d)

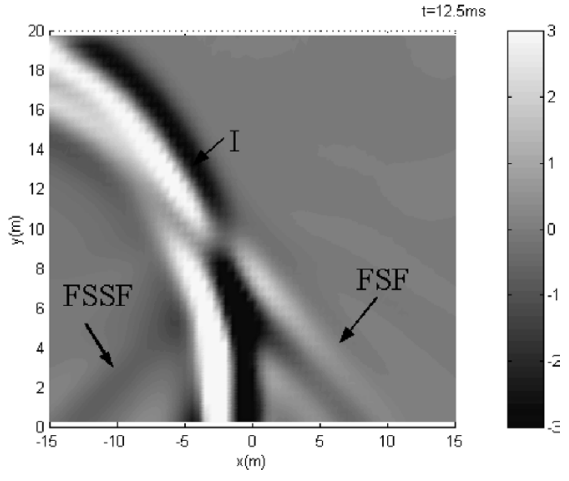

(c)

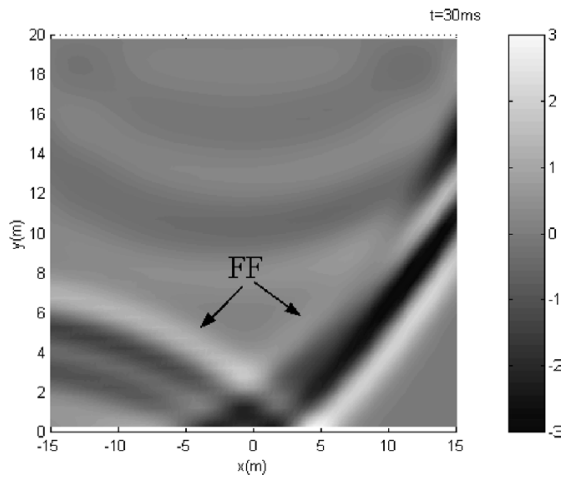

(e)

Fig. 5. Time pressure responses for a characteristic frequency of $350 \mathrm{~Hz}$, when the solid is a fast formation, registered: (a) at the lower line of receivers; over the grid of receivers at: (b) $t=7.5 \mathrm{~ms}$; (c) $t=12.5 \mathrm{~ms}$; (d) $t=20 \mathrm{~ms}$; (e) $t=30 \mathrm{~ms}$. 


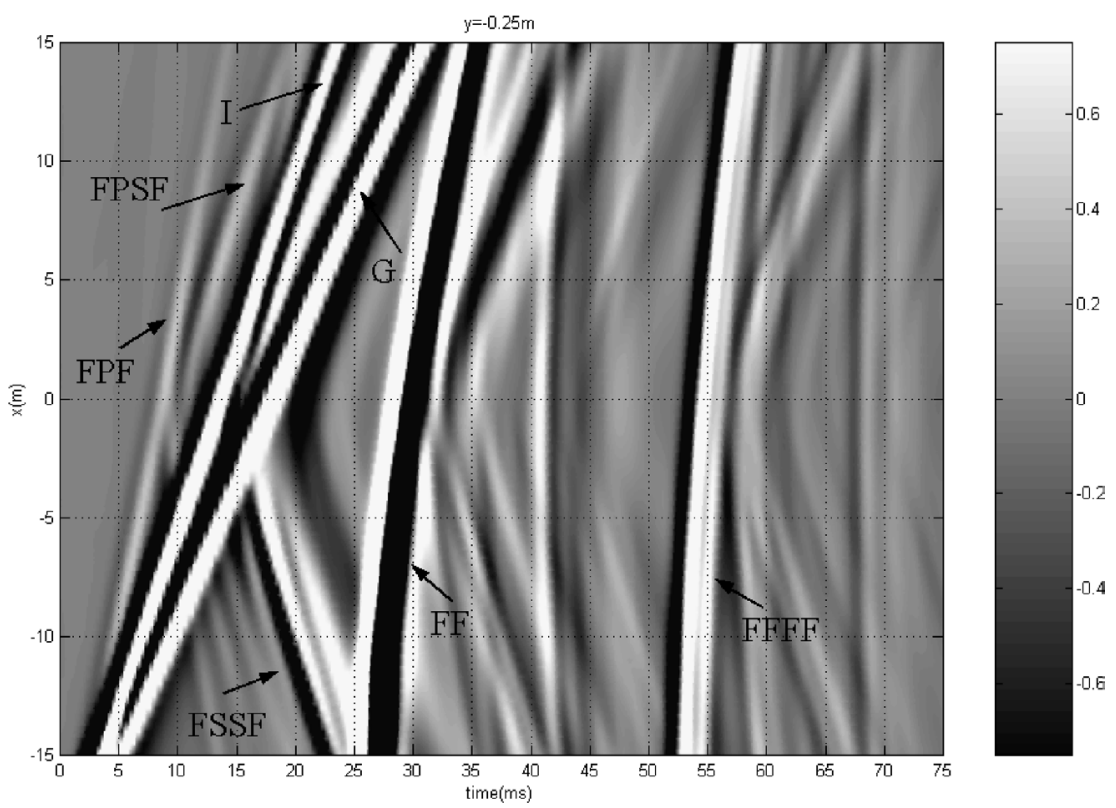

(a)

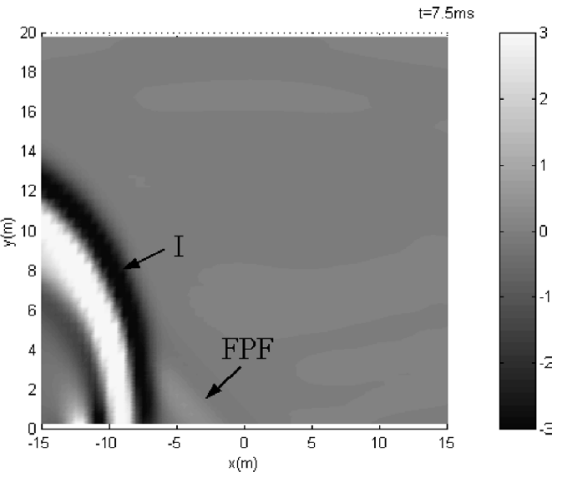

(b)

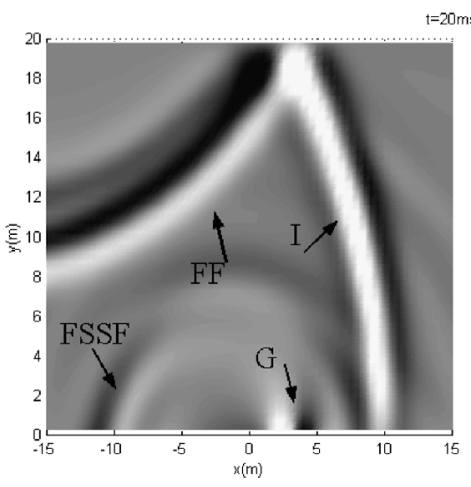

(d)

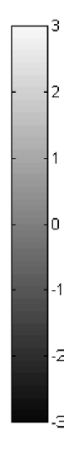

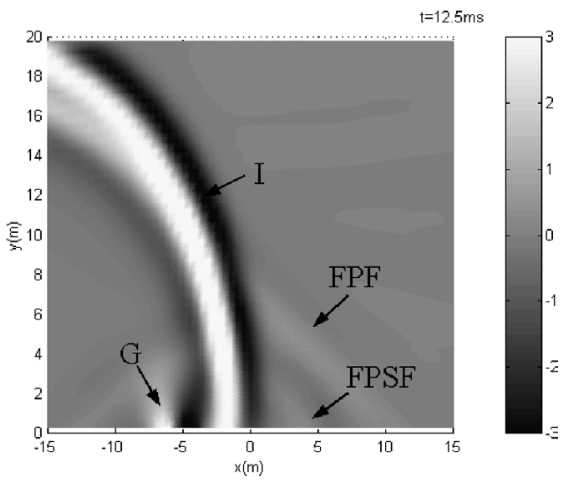

(c)

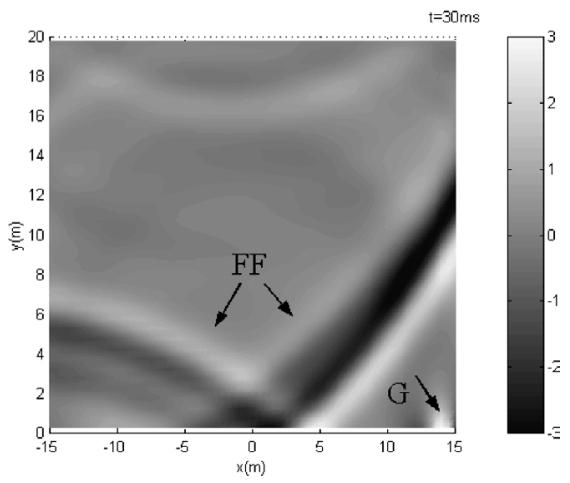

(e)

Fig. 6. Time pressure responses for a characteristic frequency of $350 \mathrm{~Hz}$, when the solid is a slow formation, registered: (a) at the lower line of receivers; over the grid of receivers at: (b) $t=7.5 \mathrm{~ms}$; (c) $t=12.5 \mathrm{~ms}$; (d) $t=20 \mathrm{~ms}$; (e) $t=30 \mathrm{~ms}$. 

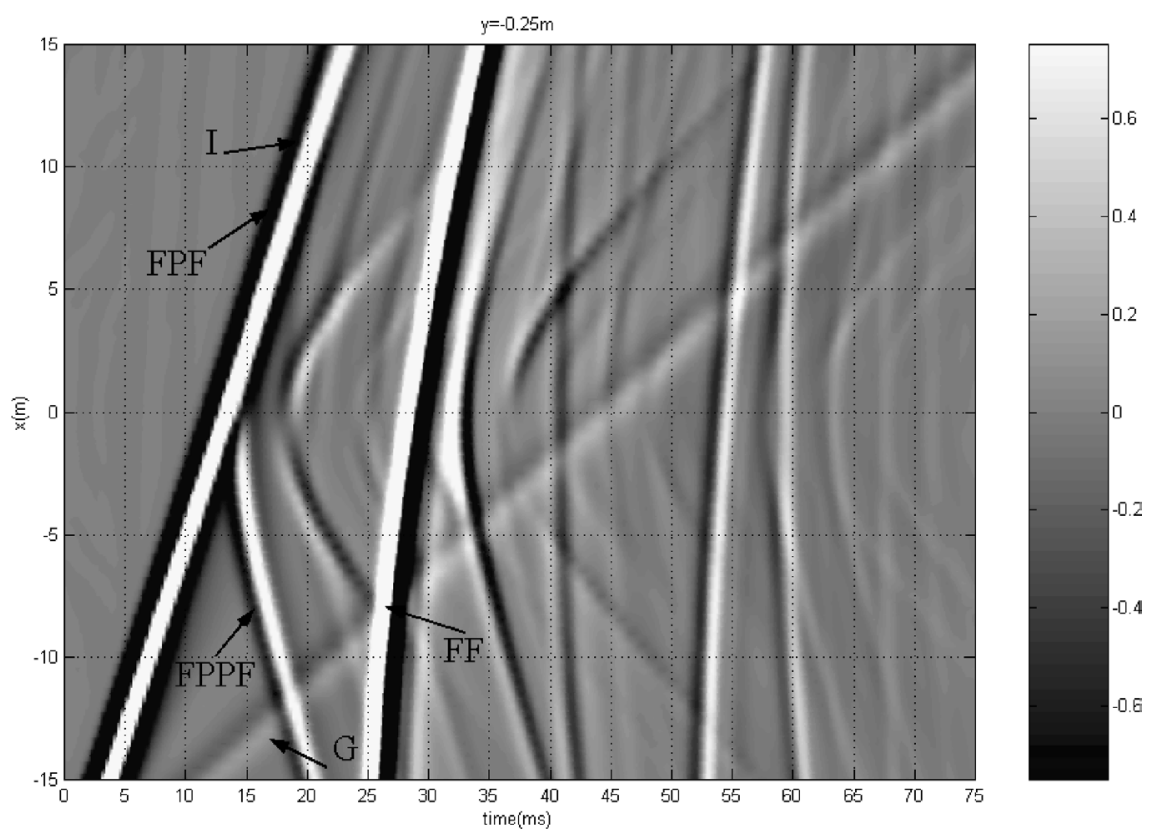

(a)

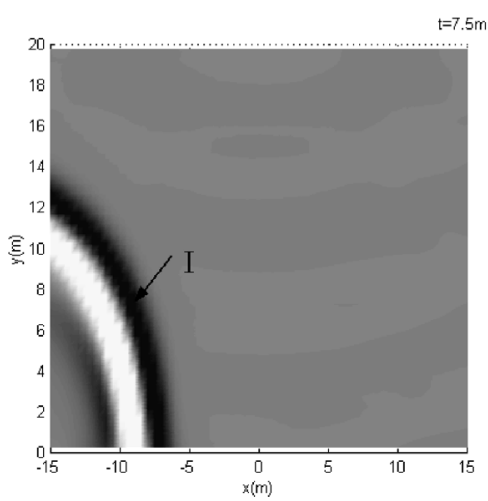

(b)

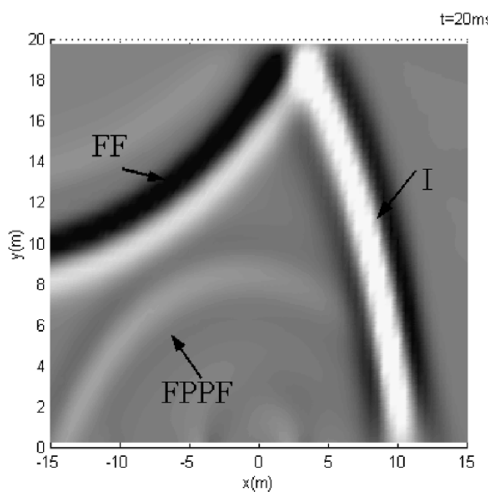

(d)

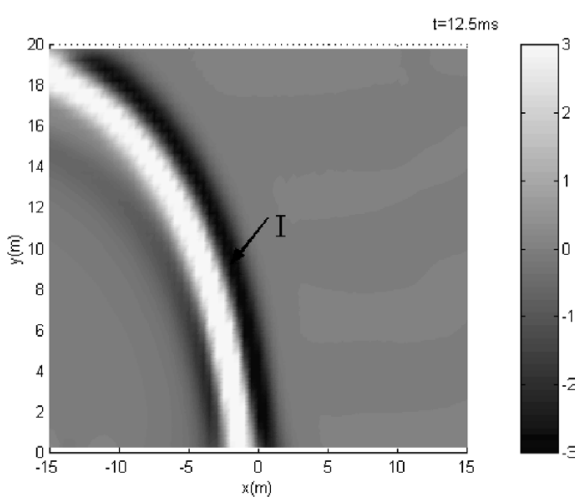

(c)

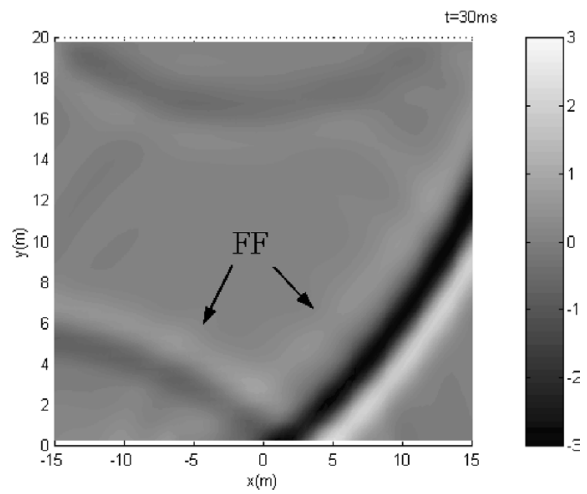

(e)

Fig. 7. Time pressure responses for a characteristic frequency of $350 \mathrm{~Hz}$, when the solid is a sediment formation, registered: (a) at the lower line of receivers; over the grid of receivers at: (b) $t=7.5 \mathrm{~ms}$; (c) $t=12.5 \mathrm{~ms}$; (d) $t=20 \mathrm{~ms}$; (e) $t=30 \mathrm{~ms}$. 
A pulse with a slight amplitude appears at $t=10 \mathrm{~ms}$ corresponding to guided waves $(\mathrm{G})$ travelling along the solid-fluid interface with velocities lower than the $\mathrm{S}$ wave velocity. Some waves propagating in the fluid medium are reflected between the top and bottom of the fluid layer. The first reflection in the top has a high amplitude and is labelled FF in the plots. High order reflections in the boundary of the rigid inclusion buried in the solid medium are also visible in Fig. 7(a).

\section{Conclusions}

The analytical functions presented in this paper can be used by themselves to compute the wave field generated by a spatially sinusoidal harmonic load in a formation composed of a fluid layer over a semi-infinite solid.

In this paper, they were also found to be useful when incorporated in a BEM formulation to compute the wavefield produced by rigid inclusions buried in a semi-infinite solid under a fluid layer.

The verification of the solutions against those provided by a BEM model using Green's functions for an unbounded medium showed a very good agreement between the two solutions. However, the proposed model can overcome the computer limitations of the former model, given that it is only necessary to discretize the boundary of the inclusions.

A fluid layer over a semi-infinite solid formation, where a rigid circular inclusion is buried, is used to illustrate the applicability of the proposed BEM models.

In all the simulations it was possible to detect different pulses resulting from reflections in the horizontal boundaries, reflections in the boundary of the inclusion and also guided waves travelling along the solid-fluid interface.

When the solid is a sediment the reflections have lower amplitude than in the other cases. This is due to the fact that much more energy is transmitted to the solid medium.

The interpretation of the results agrees with the findings obtained when the travel paths are calculated, following a ray in the wave front. It can thus be confirmed that the wave scattering produced by buried rigid inclusions in a seabed formation can be identified by sources placed in the fluid medium.

Appendix I. Definition of $\left(a_{i j}^{x}, i=1,5 ; j=1,5\right),\left(c_{i}^{x}, i=1,5\right)$ and $\left(b_{i}^{x}, i=1,5\right)$

$a_{i j}^{x}, i=1,5 ; j=1,5$,

$$
\begin{aligned}
& a_{11}^{x}=-2 k_{n}^{2}, \quad a_{12}^{x}=-k_{z}^{2}, \quad a_{13}^{x}=k_{n}^{2}-\gamma_{n}^{2}, \quad a_{14}^{x}=0, \quad a_{15}^{x}=0, \\
& a_{21}^{x}=-2, \quad a_{22}^{x}=1, \quad a_{23}^{x}=1, \quad a_{24}^{x}=0, \quad a_{25}^{x}=0, \\
& a_{31}^{x}=\frac{-k_{s}^{2}}{v_{n}}-\frac{2 v_{z n}^{2}}{v_{n}}, \quad a_{32}^{x}=0, \quad a_{33}^{x}=2 \gamma_{n}, \quad a_{34}^{x}=\frac{\mathrm{i} 2 \rho \omega^{2}}{v_{n}^{f} \mu}, \quad a_{35}^{x}=\frac{\mathrm{i} 2 \rho \omega^{2}}{\mu v_{n}^{f}} E_{f h}, \\
& a_{41}^{x}=-\mathrm{i}, \quad a_{42}^{x}=0, \quad a_{43}^{x}=\mathrm{i}, \quad a_{44}^{x}=\frac{2 \rho \omega^{2}}{k_{p_{f}}^{2} \lambda_{f}}, \quad a_{45}^{x}=-\frac{2 \rho \omega^{2}}{k_{p_{f}}^{2} \lambda_{f}} E_{f h}, \\
& a_{51}^{x}=0, \quad a_{52}^{x}=0, \quad a_{53}^{x}=0, \quad a_{54}^{x}=\frac{-\mathrm{i} 2 \rho \omega^{2}}{v_{n}^{f}} E_{f h}, \quad a_{55}^{x}=\frac{-\mathrm{i} 2 \rho \omega^{2}}{v_{n}^{f}} .
\end{aligned}
$$

$c_{i}^{x}, i=1,5$,

$$
c_{1}^{x}=A_{n}^{x}, \quad c_{2}^{x}=B_{n}^{x}, \quad c_{3}^{x}=C_{n}^{x}, \quad c_{4}^{x}=D_{n}^{x} / k_{n}, \quad c_{5}^{x}=E_{n}^{x} .
$$

$b_{i}^{x}, i=1,5$,

$$
\begin{aligned}
& b_{1}^{x}=-2 k_{n}^{2} E_{b 1}+\left(-k_{s}^{2}+2 k_{n}^{2}\right) E_{c 1}, \quad b_{2}^{x}=-2 E_{b 1}+2 E_{c 1}, \quad b_{3}^{x}=\left(\frac{k_{s}^{2}}{v_{n}}+\frac{2 v_{z n}^{2}}{v_{n}}\right) E_{b 1}-2 \gamma_{n} E_{c 1}, \\
& b_{4}^{x}=-\mathrm{i} E_{b 1}+\mathrm{i} E_{c 1}, \quad b_{5}^{x}=-\mathrm{i} E_{b 1}+\mathrm{i} E_{c 1}
\end{aligned}
$$

with $E_{b 1}=\mathrm{e}^{-\mathrm{i} v_{n}\left|-y_{0}\right|}, E_{c 1}=\mathrm{e}^{-\mathrm{i} \gamma_{n}\left|-y_{0}\right|}, v_{z n}=\sqrt{-k_{z}^{2}-k_{n}^{2}}, E_{f h}=\mathrm{e}^{-\mathrm{i} v_{n}^{f}|h|}$. 
Appendix II. Definition of $\left(a_{i j}^{y}, i=1,5 ; j=1,5\right),\left(c_{i}^{y}, i=1,5\right)$ and $\left(b_{i}^{y}, i=1,5\right)$

$a_{i j}^{y}, i=1,5, j=1,5$,

$$
\begin{aligned}
& a_{11}^{y}=-2 v_{n}, \quad a_{12}^{y}=\frac{-k_{n}^{2}}{\gamma_{n}}+\gamma_{n}, \quad a_{13}^{y}=\frac{-k_{z}^{2}}{\gamma_{n}}, \quad a_{14}^{y}=0, \quad a_{15}^{y}=0, \\
& a_{21}^{y}=-2 v_{n}, \quad a_{22}^{y}=\frac{-k_{n}^{2}}{\gamma_{n}}, \quad a_{23}^{y}=\frac{-k_{z}^{2}}{\gamma_{n}}+\gamma_{n}, \quad a_{24}^{y}=0, \quad a_{25}^{y}=0, \\
& a_{31}^{y}=\left(-k_{s}^{2}-2 v_{z n}^{2}\right), \quad a_{32}^{y}=-2 k_{n}^{2}, \quad a_{33}^{y}=-2 k_{z}^{2}, \quad a_{34}^{y}=\frac{+\mathrm{i} 2 \rho \omega^{2}}{v_{n}^{f} \mu}, \quad a_{35}^{y}=a_{3 a}^{y} E_{f h}, \\
& a_{41}^{y}=-\mathrm{i} v_{n}, \quad a_{42}^{y}=\frac{-\mathrm{i} k_{n}^{2}}{\gamma_{n}}, \quad a_{43}^{y}=\frac{-\mathrm{i} k_{z}^{2}}{\gamma_{n}}, \quad a_{44}^{y}=\frac{2 \rho \omega^{2}}{k_{p_{f}}^{2} \lambda_{f}}, \quad a_{45}^{y}=-a_{44}^{y} E_{f h}, \\
& a_{51}^{y}=0, \quad a_{52}^{y}=0, \quad a_{53}^{y}=0, \quad a_{54}^{y}=-\frac{\mathrm{i} 2 \rho \omega^{2}}{v_{n}^{f}} E_{f h}, \quad a_{55}^{y}-\frac{\mathrm{i} 2 \rho \omega^{2}}{v_{n}^{f}} .
\end{aligned}
$$

$c_{i}^{y}, i=1,5$,

$$
c_{1}^{y}=A_{n}^{y}, \quad c_{2}^{y}=B_{n}^{y}, \quad c_{3}^{y}=C_{n}^{y}, \quad c_{4}^{y}=D_{n}^{y}, \quad c_{5}^{y}=E_{n}^{y} .
$$

$b_{i}^{y}, i=1,5$,

$$
\begin{array}{ll}
b_{1}^{y}=2 v_{n} E_{b 1}-\left(\frac{v_{z n}^{2}}{\gamma_{n}}+\gamma_{n}\right) E_{c 1}, & b_{2}^{y}=2 v_{n} E_{b 1}-\left(\frac{v_{z n}^{2}}{\gamma_{n}}+\gamma_{n}\right) E_{c 1}, \\
b_{3}^{y}=\left(-k_{s}^{2}-2 v_{z n}^{2}\right) E_{b 1}+2 v_{z n}^{2} E_{c 1}, & b_{4}^{y}=\mathrm{i} v_{n} E_{b 1}+\left(\frac{\mathrm{i} k_{n}^{2}}{\gamma_{n}}+\frac{\mathrm{i} k_{z}^{2}}{\gamma_{n}}\right) E_{c 1}, \quad b_{5}^{y}=0
\end{array}
$$

with $E_{b 1}=\mathrm{e}^{-\mathrm{i} v_{n}\left|-y_{0}\right|}, E_{c 1}=\mathrm{e}^{-\mathrm{i} \gamma_{n}\left|-y_{0}\right|}, v_{z n}=\sqrt{-k_{z}^{2}-k_{n}^{2}}, E_{f h}=\mathrm{e}^{-\mathrm{i} v_{n}^{f}|h|}$.

\section{Appendix III. Definition of $\left(a_{i j}^{z}, i=1,5 ; j=1,5\right),\left(c_{i}^{z}, i=1,5\right)$ and $\left(b_{i}^{z}, i=1,5\right)$}

$a_{i j}^{z}, i=1,5 ; j=1,5$,

$$
\begin{aligned}
& a_{11}^{z}=-2, \quad a_{12}^{z}=1, \quad a_{13}^{z}=1, \quad a_{14}^{z}=0, \quad a_{15}^{z}=0, \\
& a_{21}^{z}=-2 k_{z}^{2}, \quad a_{22}^{z}=k_{z}^{2}-\gamma_{n}^{2}, \quad a_{23}^{z}=-k_{n}^{2}, \quad a_{24}^{z}=0, \quad a_{25}^{z}=0, \\
& a_{31}^{z}=-\left(\frac{k_{s}^{2}}{v_{n}}+\frac{2 v_{z n}^{2}}{v_{n}}\right), \quad a_{32}^{z}=2 \gamma_{n}, \quad a_{33}^{z}=0, \quad a_{34}^{z}=\frac{+\mathrm{i} 2 \rho \omega^{2}}{v_{n}^{f} \mu}, \quad a_{35}^{z}=a_{34}^{z} E_{f h}, \\
& a_{41}^{z}=-\mathrm{i}, \quad a_{42}^{z}=\mathrm{i}, \quad a_{43}^{z}=0, \quad a_{44}^{z}=\frac{2 \rho \omega^{2}}{k_{p_{f}}^{2} \lambda_{f}}, \quad a_{45}^{z}=-a_{44}^{z} E_{f h}, \\
& a_{51}^{z}=0, \quad a_{52}^{z}=0, \quad a_{53}^{z}=0, \quad a_{54}^{z}=-\frac{\mathrm{i} 2 \rho \omega^{2}}{v_{n}^{f}} E_{f h}, \quad a_{55}^{z}=-\frac{\mathrm{i} 2 \rho \omega^{2}}{v_{n}^{f}} .
\end{aligned}
$$

$c_{i}^{z}, i=1,8$,

$$
c_{1}^{z}=A_{n}^{z}, \quad c_{2}^{z}=B_{n}^{z}, \quad c_{3}^{z}=C_{n}^{z}, \quad c_{4}^{z}=D_{n}^{z}, \quad c_{5}^{z}=E_{n}^{z} .
$$

$b_{i}^{z}, i=1,5$,

$$
\begin{aligned}
& b_{1}^{z}=2\left(-E_{b 1}+E_{c 1}\right), \quad b_{2}^{z}=-2 k_{z}^{2} E_{b 1}+\left(k_{z}^{2}-\gamma_{n}^{2}-k_{n}^{2}\right) E_{c 1}, \\
& b_{3}^{z}=\left(\frac{k_{s}^{2}}{v_{n}}+\frac{2 v_{z n}^{2}}{v_{n}}\right) E_{b 1}-2 \gamma_{n} E_{c 1}, \quad b_{4}^{z}=-\mathrm{i} k_{z} E_{b 1}+\mathrm{i} k_{z} E_{c 1}, \quad b_{5}^{z}=0
\end{aligned}
$$

with $E_{b 1}=\mathrm{e}^{-\mathrm{i} v_{n}\left|-y_{0}\right|}, E_{c 1}=\mathrm{e}^{-\mathrm{i} \gamma_{n}\left|-y_{0}\right|}, v_{z n}=\sqrt{-k_{z}^{2}-k_{n}^{2}}, E_{f h}=\mathrm{e}^{-\mathrm{i} v_{n}^{f}|h|}$. 
Appendix IV. Definition of $\left(a_{i j}^{f}, i=1,5 ; j=1,5\right),\left(c_{i}^{f}, i=1,5\right)$ and $\left(b_{i}^{f}, i=1,5\right)$

$$
\begin{aligned}
& a_{i j}^{f}, i=1,5 ; j=1,5, \\
& a_{11}^{f}=-2 v_{n}, \quad a_{12}^{f}=\frac{-k_{n}^{2}}{\gamma_{n}}+\gamma_{n}, \quad a_{13}^{f}=\frac{-k_{z}^{2}}{\gamma_{n}}, \quad a_{14}^{f}=0, \quad a_{15}^{f}=0, \\
& a_{21}^{f}=-2 v_{n}, \quad a_{22}^{f}=\frac{-k_{n}^{2}}{\gamma_{n}}, \quad a_{23}^{f}=\frac{-k_{z}^{2}}{\gamma_{n}}+\gamma_{n}, \quad a_{24}^{f}=0, \quad a_{25}^{f}=0, \\
& a_{31}^{f}=-k_{s}^{2}-2 v_{z n}^{2}, \quad a_{32}^{f}=-2 k_{n}^{2}, \quad a_{33}^{f}=-2 k_{z}^{2}, \quad a_{34}^{f}=\frac{-\mathrm{i} 2 \rho \omega^{2}}{v_{n}^{f} \mu}, \quad a_{35}^{f}=a_{34}^{y} E_{f h}, \\
& a_{41}^{f}=-\mathrm{i} v_{n}, \quad a_{42}^{f}=\frac{-\mathrm{i} k_{n}^{2}}{\gamma_{n}}, \quad a_{43}^{f}=\frac{-\mathrm{i} k_{z}^{2}}{\gamma_{n}}, \quad a_{44}^{f}=\frac{2 \rho \omega^{2}}{k_{p_{f}}^{2} \lambda_{f}}, \quad a_{45}^{f}=-a_{44}^{f} E_{f h}, \\
& a_{51}^{f}=0, \quad a_{52}^{f}=0, \quad a_{53}^{f}=0, \quad a_{54}^{f}=\frac{-\mathrm{i} 2 \rho \omega^{2}}{v_{n}^{f}} E_{f h}, \quad a_{55}^{f}=\frac{-\mathrm{i} 2 \rho \omega^{2}}{v_{n}^{f}} .
\end{aligned}
$$

$c_{i}^{f}, i=1,5$,

$$
c_{1}^{f}=A_{n}^{f}, \quad c_{2}^{f}=B_{n}^{f}, \quad c_{3}^{f}=C_{n}^{f}, \quad c_{4}^{f}=D_{n}^{f}, \quad c_{5}^{f}=E_{n}^{f} .
$$

$b_{i}^{f}, i=1,5$,

$$
b_{1}^{f}=0, \quad b_{2}^{f}=0, \quad b_{3}^{f}=\frac{-\mathrm{i} 2 \rho \omega^{2}}{v_{n}^{f} \mu} E_{f 1}, \quad b_{4}^{f}=\frac{2 \rho \omega^{2}}{k_{p f}^{2} \lambda_{f}} E_{f 1}, \quad b_{5}^{f}=\frac{2 \mathrm{i} \rho \omega^{2}}{v_{n}^{f}} E_{f h 1}
$$

with $v_{z n}=\sqrt{-k_{z}^{2}-k_{n}^{2}}, E_{f h}=\mathrm{e}^{-\mathrm{i} v_{n}^{f}|h|}, E_{f 1}=\mathrm{e}^{-\mathrm{i} v_{n}^{f}\left|y_{0}\right|}, E_{f h 1}=\mathrm{e}^{-v_{n}^{f}\left|h+y_{0}\right|}$.

\section{References}

Boström, A., Kristensson, G., 1980. Elastic wave scattering by a three-dimensional inhomogeneity in an elastic half space. Wave Motion 2, 335-353.

Bouchon, M., 1979. Discrete wave number representation of elastic wave fields in three-space dimensions. J. Geophys. Res. 84, $3609-3614$.

Branco, F., Godinho, L., Tadeu, A., 2002. Propagation of pressure waves inside a confined fluid channel with an irregular floor. J. Comput. Acoustics 10, 183-194.

Datta, S.K., Shah, A.H., 1982. Scattering of SH-waves by embedded cavities. Wave Motion 4, 265-283.

Davis, C.A., Lee, V.W., Bardet, J.P., 2001. Transverse response of underground cavities and pipes to incident SV waves. Earthquake Engrg. Struct. Dynamics 30, 383-410.

Dawson, T., Fawcett, J., 1990. A boundary integral equation method for acoustic scattering in a waveguide with nonplanar surfaces. J. Acoust. Soc. Am. 87, 1110-1125.

Fawcett, J., 1996a. The computation of the scattered pressure field from a cylinder embedded between two half-spaces with different densities. J. Acoust. Soc. Am. 99, 2435-2438.

Fawcett, J., 1996b. Acoustic scattering from cylindrical objects embedded between two half-spaces. J. Acoust. Soc. Am. 100, $3053-3060$.

Fawcett, J., Dawson, T., 1990. Fourier synthesis of three-dimensional scattering in a two-dimensional oceanic waveguide using boundary integral equation methods. J. Acoust. Soc. Am. 88, 1913-1920.

Godinho, L., Tadeu, A., Branco, F., 2001. 3D acoustic scattering from an irregular fluid waveguide via the BEM. EABE - Engineering Analysis with Boundary Elements J. 25, 443-453.

Guzina, B.B., Fataa, S.N., 2003. On the stress-wave imaging of cavities in a semi-infinite solid. Int. J. Solids Structures 40, $1505-1523$.

Hackman, R., Sammelmann, G., 1986. Acoustic scattering in an inhomogeneous waveguide: theory. J. Acoust. Soc. Am. 80, $1447-1458$.

Ingenito, F., 1987. Scattering from an object in a stratified medium. J. Acoust. Soc. Am. 82, 2051-2059.

Kim, J., Papageorgiou, A.S., 1993. Discrete wavenumber boundary element method for 3-D scattering problems. J. Engrg. Mech. ASCE 119, 603-624.

Kristensson, G., Ström, S., 1978. Scattering from buried inhomogeneities - a general three-dimensional formalism. J. Acoust. Soc. Am. 64, 917-936.

Lamb, H., 1904. On the propagation of tremors at the surface of an elastic solid. Philos. Trans. Roy. Soc. London Ser. A $203,1-42$.

Lee, V.W., 1977. On deformations near circular underground cavity subjected to incident plane SH waves. In: Symp. of Appl. Computer Methods in Engrg., Univ. of Southern California, Los Angeles, pp. 951-961. 
Lee, V.W., 1988. Three-dimensional diffraction of elastic waves by a spherical cavity in an elastic half-space. 1: Closed-form solutions. Soil Dynamics Earthquake Engrg. 7, 149-161.

Lee, V.W., Karl, J.A., 1992. Diffraction of SV waves by underground circular cylindrical cavities. Soil Dynamics Earthquake Engrg. 11, 445456.

Lim, R., 1998. Acoustics scattering by a partially buried three-dimensional elastic obstacle. J. Acoust. Soc. Am. 104, 769-782.

Lim, R., Lopes, J., Hackman, R., Todoroff, D., 1993. Scattering by objects buried in underwater sediments: theory and experiment. J. Acoust. Soc. Am. 93, 1762-1782.

Makris, N.C., 1998. A spectral approach to 3D object scattering in layered media applied to scattering from submerged spheres. J. Acoust. Soc. Am. 104, 2105-2113.

Sammelmann, G., Hackman, R., 1987. Acoustic scattering in an homogeneous waveguide. J. Acoust. Soc. Am. 82, 324-336.

Tadeu, A.J.B., Kausel, E., 2000. Green's functions for two-and-a-half dimensional elastodynamic problems. J. Engrg. Mech. ASCE 126, 10931097. 